\title{
Obstructive sleep apnea and hormones - a novel insight
}

Marek Ruchała ${ }^{1}$, Barbara Bromińska ${ }^{1}$, Ewa Cyrańska-Chyrek ${ }^{1}$, Barbara Kuźnar-Kamińska ${ }^{2}$, Magdalena Kostrzewska², Halina Batura-Gabryel ${ }^{2}$

${ }^{1}$ Department of Endocrinology, Metabolism and Internal Medicine, Poznan University of Medical Sciences, Poznan, Poland

2Department of Pulmonology, Allergology and Respiratory Oncology,

Poznan University of Medical Sciences, Poznan, Poland

Submitted: 24 January 2016

Accepted: 7 June 2016

Arch Med Sci 2017; 13, 4: 875-884

DOI: https://doi.org/10.5114/aoms.2016.61499

Copyright $\odot 2016$ Termedia \& Banach

\section{Abstract}

Obstructive sleep apnea (OSA), a disorder characterized by repetitive collapse of the upper respiratory tract during sleep, occurs in about $4 \%$ of middle-aged men and $2 \%$ of women. The incidence of the disorder is rising due to an increase in obesity and ageing of the population. Patients with obstructive sleep apnea are at elevated risk of some endocrinal and metabolic disorders, which may lead to serious consequences including shortening of life expectancy. The recognition and understanding of interactions between local upper airway dysfunction and its endocrinal consequences is therefore vital. In this review we will focus on the influence of OSA on bone metabolism and endocrine homeostasis.

Key words: inflammation, sleep apnea, hormone, metabolic disorders, bones.

\section{Introduction}

Repetitive collapse of the upper respiratory tract during sleep is a feature of the disorder called obstructive sleep apnea (OSA). Periods of physiological sleep are disturbed by arousals related to respiratory effort, apneas or hypopneas. The patient usually complains about day time sleepiness, fatigue, lack of concentration and awakenings with a sensation of choking or heart pain. Family members notice snoring and breaks in breathing while sleeping [1]. Obstructive sleep apnea occurs in about $4 \%$ of middle-aged men and $2 \%$ of women [2]. The incidence of the disorder is rising due to an increase in obesity and ageing of the population. Long-term consequences of OSA include impaired cognitive function, insulin resistance, cardiovascular diseases and depression. It contributes to increased morbidity, mortality and health costs [3]. In this review we aim to address the issue of the influence of OSA on bone metabolism and endocrine homeostasis (Figure 1).

\section{Obstructive sleep apnea and bones}

Disturbance in sleep patterns influence bone metabolism. Obstructive sleep apnea characterized by arousals disturbing the physiological rest-wakening cycle may disrupt signals mediating bone formation, e.g. interaction between bone clock genes and the suprachiasmatic nucleus.

\author{
Corresponding author: \\ Barbara Bromińska MD \\ Department of Endocrinology, \\ Metabolism and \\ Internal Medicine \\ Poznan University \\ of Medical Sciences \\ 49 Przybyszewskiego St \\ 60-355 Poznan, Poland \\ Phone: +48 618691330 \\ Fax: +48 618691682 \\ E-mail: barbarabrominska@ \\ gmail.com
}




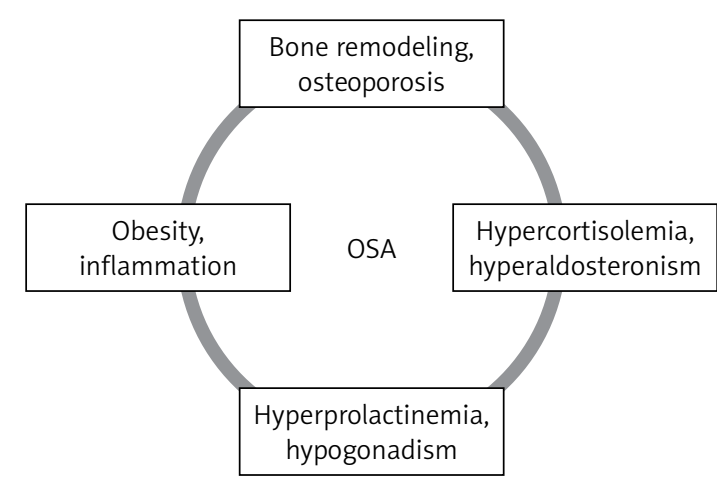

Figure 1. Endocrine disorders in the course of OSA

Bone remodeling reflected by levels of bone turnover markers in urine and serum is altered by sleep disturbance. Obstructive sleep apnea influences the skeleton in several possible mechanisms, namely by inducing hypoxia, oxidative stress and changing melatonin, leptin and vitamin D secretion. The impact of OSA on bone mineral density (BMD) - an important parameter in assessing its health, especially in the elderly - is unequivocal.

Alterations in circadian rhythm change the bone. Synchrony between the environment and organism and inside the human body is necessary to maintain homeostasis. It helps to adjust the efficacy and speed of metabolic processes to changing conditions. Disruption of this balance may cause metabolic illnesses and cancer and increase mortality [4]. Rhythmicity is also noticed in some aspects of skeletal metabolism. Signals mediating bone formation and resorption, subordinate to circadian rhythms, are transmitted to bone cells from the suprachiasmatic nucleus through direct neuronal connections, hormonal impulses and other mechanisms. The skeleton presents clock genes, e.g. Cry2, Per1, Per2, and BMAL1, in osteoclasts. Their expression varies according to bodily demands, timing and external conditions [5]. What is important, when sleep patterns are altered, as in OSA, mechanisms regulating the circadian cycle also change. To conclude, it possibly leads to desynchrony in clock gene expression and influences bone metabolism.

Bone remodeling is a continuous process of its synthesis and destruction. The bone turnover markers - substances produced during bone metabolism - are partially subordinate to day/night cyclic patterns. Thus, changes in their levels in OSA patients may reflect their role in skeleton reshaping. Present in urine and blood, these markers are usually used to monitor osteoporosis treatment. Tomiyama et al. reported that OSA is associated with increased amounts of a bone resorption marker in the urine - CTX (collagen type I crosslinked C-telopeptide). Moreover, treatment of OSA with continuous positive airway pressure (CPAP) reduces the concentration of CTX in urine, possibly by restoration of the normal diurnal pattern, reducing bone resorption [6]. However, there are no reports of a negative influence of CPAP therapy in OSA on bones. It underlines the detrimental influence of sleep alteration on skeleton metabolism, but also gives hope that changes might be reversed by adequate therapy.

Bone reshaping is affected by hypoxia and oxidative stress. Normally, low oxygen concentrations in bone tissue occur during maturation and after fractures. Pathologic intermittent hypoxia characteristic for OBS creates an acidotic environment and inflammation in the skeleton associated with fractures. Obstructive sleep apnea is also associated with systemic inflammation. The Health, Ageing and Body Composition Study shows that, in subjects with high inflammation markers, the risk of fracture is markedly elevated in comparison to a group with those parameters within the normal range [7]. Recently published studies have reported reduction of those substances after CPAP treatment [8]. Moreover, deficiency of oxygen causes oxidative stress, which is characterized by excessive bioavailability of reactive oxygen species $[9,10]$. It increases the possibility of fracture and osteoporosis. Obstructive sleep apnea is possibly associated with decreased total plasma antioxidant status and increased production of free radicals [11]. When bone breaks, even more free radicals are generated, finally causing lipid peroxidation. Additionally, old osteoporotic patients are deficient in antioxidative vitamins, which further worsens the condition of the tissue [12]. To sum up, hypoxia induced by OSA leads to a chain of events, which can result in bone fracture, especially in the elderly. Continuous positive airway pressure treatment, by reducing periods of low oxygenation, may likely diminish inflammation, but its role in preventing skeletal damage is unknown.

Obstructive sleep apnea, by sleep fragmentation, stimulates the sympathetic system. Its excessive activation strains bone formation. Also leptin, known as a satiety hormone [13], indirectly increases sympathetic tone. This protein is secreted in harmony with the circadian cycle, but its levels alter due to sleep/wake and eating/fasting patterns [14]. Due to the different sleep cycle in OSA, the leptin concentration changes. Its levels increase, and decrease after CPAP treatment [15].

Melatonin is a hormone released by the pineal gland, known for its regulatory role in the night/ day cycle. Due to frequent arousals during the night, patients with OSA are exposed to more abnormal nocturnal light, which affects melatonin secretion. Melatonin acts as an antioxidant, reducing the formation of free radicals in bone cells in in-vitro studies. Moreover, this peptide pro- 
motes human mesenchymal stem cells to become osteoblastic precursors. In animal models, melatonin has been shown to have a positive influence on bone metabolism [16]. In a recently published study by Amstrup, 1-year treatment with melatonin in osteopenic postmenopausal women increased BMD at the femoral neck in a dose-dependent manner. Thus, it did not change markers of bone turnover [17]. A small study by Zirlik et al. showed that melatonin peak time occurred later than usual in OSA patients (6:00 AM). Short treatment with CPAP (3 months) normalizes secretion cycles [18]. Obstructive sleep apnea by increasing leptin secretion might be associated with promotion of bone resorption. What is more, sleep disturbances induce alteration in the melatonin cycle, depriving bones of its beneficial effects, which was described both in animals and humans. Continuous positive airway pressure therapy can normalize both leptin and melatonin levels and possibly reverse destruction of the bones. It is an additional advantage of such treatment.

Obstructive sleep apnea may affect bone also by reducing vitamin $D$ levels. This steroid is indispensable not only in skeleton metabolism, but was claimed to have anti-cancer and anti-inflammatory properties. Bozkurt et al. observed lower vitamin D levels in OSA patients. The stage of deficiency correlated with the severity of the breathing disorder and predisposition to glucose metabolism impairment [19]. A study on the influence of CPAP in OSA on vitamin D concentration proved that short treatment (7 days) increased levels of this substance, but only in men [20]. Obstructive sleep apnea by decreasing vitamin $\mathrm{D}$, besides an adverse effect on bony structure, impairs its desirable anti-inflammatory and neoplasm preventing role. This underlines even more the importance of CPAP in OSA.

Bone mineral density, which is used to assess bone structure, could be influenced by sleep disturbance. Bone mineral density indirectly gives an insight into the condition of the skeleton and is an indicator of osteoporosis and risk of fracture. Studies on the influence of OSA on BMD gave conflicting results. Sforza et al. demonstrated that proximal lumbar and femur BMD values correlate positively with the severity of nocturnal hypoxemia, suggesting a protective influence of low oxygen concentration on bone. The authors suggested that chronic intermittent hypoxia stimulates human mesenchymal stem cells to differentiate into osteoblasts [21]. On the other hand, Wang et al. examined 66 patients with chronic obstructive pulmonary disease (COPD). In subjects with concomitant OBS, bone mineral density was significantly lower than in controls [22]. Song et al. mimicked OSA in ovariectomized rats. They found that hypoxia can reduce mineral density and alter bone architecture. The authors applied genistein (phytoestrogen) after the exposure to hypoxia. This intervention increased bone mineral density and expression of osteogenesis-related genes and modified bone architecture. It suggests that phytoestrogens and estrogen-like drugs may be considered in the future as treatment for OSA postmenopausal women [23]. The knowledge of the influence of OSA on BMD is incomplete. It can either destroy or restore bony structure. Further studies are needed to clarify this issue.

Sleep disruption might be associated with higher incidence of fracture. Both sleep and bone disorders are more prevalent in the elderly. Disturbance in diurnal rest-wakefulness rhythms leads to daytime sleepiness and impaired cognition, thus increasing the risk of falling and fracture. Chen et al. found that the risk of osteoporosis is higher in patients diagnosed with OSA in comparison to age-matched controls [24]. Cauley et al. found that hypoxia (percentage of sleep time with arterial oxygen saturation less than $90 \%$ measured using polysomnography) during sleep may be associated with greater incidence of falling and non-spine fracture in patients who presented with a low oxygen concentration for $10 \%$ or more of sleep time [25]. To summarize, OSA may increase the risk of osteoporosis and fracture. Both of those entities are connected with poorer life quality and raised expenses on healthcare. Hence, it further stresses the importance of the covered topic.

\section{Obstructive sleep apnea and gonads}

Sex hormones and breathing affect each other mutually. It is not clarified which hormone is crucial, but progesterone, androgens and estrogens are clearly indicated in the process. On the other hand, sleep disorders, such as OSA, alter hormonal homeostasis. Due to different respiration control and hormones affecting female and male bodies, we reflect separately on the influence of OSA on both genders.

Firstly, we provide a general overview of how sex hormones impact breathing. Neurosteroids are synthesized in the whole human nervous system from cholesterol, steroid precursors and sex steroids circulating in the blood stream. They indirectly modulate breathing through gamma-aminobutyric acid (GABA) or N-methyl-D-aspartate (NMDA) signaling pathways. According to animal studies, the influence may be immediate through androgen, progesterone and estradiol receptors present in the nervous system (carotid body, brainstem). The regulation of breathing is also attributed to brainstem serotoninergic neurons. The structure and activity of all these mechanisms is sex-dependent [26]. It is not clear which hormone 
is crucial in breathing. Progesterone is associated with hyperventilation during pregnancy. In mice knocked down for the progesterone receptor, the reaction to hypoxia is reduced [27]. Driver et al. found that upper respiratory tract resistance in women is lower in the luteal phase of the menstrual cycle than in the follicular one [28]. These data suggest a positive effect of progesterone on airways. In post-menopausal women on estrogen therapy the incidence of sleep-associated breathing disorders is lower than in treatment-naive women. The connection is even stronger in subjects on estrogen-progesterone therapy, indicating the synergistic effect of those hormones [29]. Studies on the influence of testosterone on respiration have provided conflicting results. Zabka et al. suggested that testosterone impacts breathing via estrogens [30]. To sum up, there are many mechanisms in which hormones affects respiration, but all those regulatory mechanisms are sex-dependent.

Sleep disturbance possibly alter hormonal levels and sexual behavior. Testosterone is secreted in a cyclic manner, mostly due to sleep patterns, and not circadian rhythm. Its concentration peaks in the blood during sleep in the first REM phase and reaches its nadir in the afternoon. Three hours of sleep with normal architecture should be sufficient to maintain testosterone secretion. Additionally, testosterone is subordinated to $\mathrm{LH}$ pulses which occur every 90 min [31]. Hence, sleep disruption may be associated with hormonal imbalance. In a study on male rodents, sleep deprivation led to lower testosterone levels and worse sperm viabilities than in controls [32]. JauchChara et al. assessed the hormonal reaction to total sleep deprivation in healthy men. Total testosterone and prolactin levels were significantly lower in the sleep-deficient group [33].

While data on sleep deprivation are quite consistent, results from research on sleep restriction are contradictory. In young healthy males after a week of $5 \mathrm{~h}$ of sleep per night testosterone levels during the day decreased by $10-15 \%$. These men also complained of low libido and vitality [34]. On the other hand, in a similarly constructed study, another group of authors did not find changes in testosterone after sleep reduction to $4 \mathrm{~h}$ for 5 nights [35]. The influence of sleep disturbance on testosterone may be due not only to limitation of rest time, but also to the part of the night when this occurs [36]. While there are plenty of studies on males concerning hormones and sleep, in women the subject has been largely overlooked. Kalmbach et al. investigated the influence of night sleep duration and quality on female sexual function. They concluded that sufficient night rest is indispensable to achieve sexual desire and proper genital response [37]. What is most important, sleep deprivation in men lowers testosterone levels, while the effect of restriction is not clear. Women respond to poor night rest with decreased sexual desire. It points to a conclusion that sleep is vital in human reproduction and sexual life.

Studies on the effect of OSA on the male gonadal axis are incompatible. Disrupted sleep architecture may impede physiological nocturnal testosterone rises. In a paper discussing the effect of OSA on the gonadal axis Luboshitzky et al. found a significant negative correlation between the respiratory distress index and LH-testosterone profiles. They suggested that sleep fragmentation, in addition to a high body mass index (BMI) index and ageing, is responsible for hypogonadism in OSA patients [38]. Gambineri et al. tested 15 abdominally obese males with metabolic syndrome and OSA, and compared the results with age- and anthropometric parameter-matched controls without sleep disorders. Both total and free testosterone levels were lower in OSA males. Additionally, negative correlations between polysomnographic parameters and testosterone were found. The number of desaturations per sleeping hour correlated adversely with total and free testosterone even after adjusting for BMI and waist values. This indicates that the severity of hypoxia may influence the gonadal axis independently of obesity [39]. In contrast, Barrett-Connor et al. described the relationships between decreased total testosterone levels, poorer sleep quality and desaturations, but these associations were attenuated or disappeared after correction for BMI and waist size [40].

Also, the influence of treatment of OSA with CPAP therapy on male gonadal axis is controversial. A recently published meta-analysis including 7 studies did not reveal any changes in total, free testosterone and sex hormone binding globulin (SHBG) before and after therapy, irrespective of treatment time [41]. Obstructive sleep apnea, as well as hypogonadism, leads to fatigue and reduced activity, decreasing the quality of life. Testosterone deficiency in OSA patients was shown to be an independent predictor of fatigue in OSA males [42]. Testosterone therapy in OSA patients might reduce such symptoms, but its usage is still under debate. The effect of a hormone may be dependent on the given dose: therapeutic or supraphysiological. Both testosterone deficiency and large doses of exogenous hormone adversely influence respiration. There have been studies suggesting that testosterone therapy may aggravate or even induce OSA [43].

It must be stressed that studies on the OSA influence on male hormonal homeostasis give conflicting results. Treatment with CPAP has an uncertain impact on testosterone levels. On the other hand, testosterone supplementation in deficient OSA patient should be introduced only with great consideration. It raises a question of proper 
medical care in such cases and creates a need for further studies.

While studies on OSA and gonadal function in men are abundant, in women knowledge of this topic is scant. Subramanian et al. assessed sexual dysfunction (SD) with the Female Sexual Function Index (FSFI) in a small group of premenopausal women with OSA. Sexual dysfunction was significantly more prevalent in subjects suffering from sleep disorders [44]. These data were supported by Petersen et al., who investigated 80 female patients varying in age. They did not observe an association between the severity of the disease and SD [45]. More recently, research on both pre- and post-menopausal women was conducted. The authors assessed not only SD but also hormonal changes in women with OSA. In both groups the FSFI score was lower than in controls. There was also a correlation between the severity of OSA and SD, even after adjusting for BMI. Additionally, in the pre-menopausal group progesterone levels correlated negatively with FSFI scores. Progesterone and estradiol levels were lower in severe OSA than in controls. The authors concluded that women with severe OSA have worse sexual function than healthy ones [46]. However, CPAP therapy (long or short term) did not affect female hormonal status [47].

Polycystic ovary syndrome (PCOS) is the most common endocrinopathy among young women. It is associated with increased risk of metabolic disorders and the possibility of developing OSA. This breathing disorder has been shown to be more prevalent in pre-menopausal PCOS women than in controls, even when taking into account BMI [48]. Conversely, Mokhlesi et al. assembled a group of obese and non-obese PCOS patients and compared them to obese and non-obese controls. All subjects completed Berlin questionnaires - a useful tool to assess risk of OSA. Polysomnography was not performed. The authors found that risk of OSA was elevated in PCOS patients, but only in obese ones. They concluded that obesity is probably a risk factor for OSA [49].

Although the problem of OSA and hormonal homeostasis in women has been neglected, we may conclude from existing studies that OSA negatively influences sexual function as well as progesterone and estradiol levels. In young PCOS women incidence of this sleep disturbance was high, so they can be subjected to its long-term consequences, e.g. insulin resistance, cardiovascular diseases and depression. These issues require more attention in the future (Table I).

\section{Obstructive sleep apnea and hyperprolactinemia}

Plasma prolactin (PRL) concentrations show a sleep-dependent pattern, with increased secre- tion during sleep and subsequent lower levels during wakening periods. The syndrome of OSA is associated with chronic sleep fragmentation and severe hypoxemia, both of which could affect the sleep-entrained PRL rhythm [50]. The change in serum prolactin (PRL) secretion in OSA is thought to be related to hypoxic stress [51]. Studies on the PRL secretion axis in OSA provide conflicting results. Due to current disagreements on this topic, Macrea et al. investigated whether CPAP therapy is associated with a significant decrease in serum PRL levels, and also whether, apart from PRL, any other hormone serum level (including that of $\mathrm{FSH}, \mathrm{LH}$, and testosterone) is changed significantly after the CPAP therapy [52]. Also, Bratel et al. reported a significant reduction in serum prolactin caused by CPAP treatment [53]. No correlations for PRL levels in patients with severe OSA and no changes induced by CPAP treatment were found by Meston et al. [54] or Spiegel et al. [55]. Recent studies have suggested an emerging hypothesis that sleep disorders, e.g. OSA, could also be related to miscarriages due to its coexistence with polycystic ovarian syndrome and overweight [56]. Taking into account such divergent results of previous studies about hyperprolactinemia in the course of OSA, its effects on sex hormones require further observations.

\section{Obstructive sleep apnea and hyperaldosteronism}

Obstructive sleep apnea is commonly related to hyperaldosteronism and both are usually associated with resistant hypertension. Hypoxia-induced sympathetic activation accompanies OSA and may lead to hypertension via stimulation of the renin-angiotensin-aldosterone system. Dudenbos-

Table I. Hormonal changes in OSA before and after CPAP treatment

\begin{tabular}{|lcc|}
\hline Variable & $\begin{array}{c}\text { Before CPAP } \\
\text { treatment }\end{array}$ & $\begin{array}{c}\text { After CPAP } \\
\text { treatment }\end{array}$ \\
\hline CTX & $\uparrow$ & $\downarrow$ \\
\hline Melatonin & $\uparrow$ & $\approx$ \\
\hline Leptin & $\uparrow$ & $\downarrow$ \\
\hline Vitamin D & $\uparrow$ & $\downarrow$ \\
\hline Testosterone & $\downarrow \leftrightarrow$ & $\leftrightarrow$ \\
\hline Estradiol, progesterone & $\downarrow$ & $\downarrow \leftrightarrow$ \\
\hline Prolactin (PRL) & $\uparrow \leftrightarrow$ & $\leftrightarrow \downarrow$ \\
\hline Aldosterone & $\uparrow$ & $\downarrow$ \\
\hline Insulin & $\uparrow$ & $\downarrow \leftrightarrow$ \\
\hline Cortisol & $\uparrow \leftrightarrow$ & $\leftrightarrow$ \\
\hline
\end{tabular}

$\uparrow$ increase, $\downarrow$ decrease, $\leftrightarrow$ without change, $\approx$ change. 
tel et al. suggest that untreated OSA is strongly associated with an increased risk of prevalent hypertension and also that OSA may increase the risk of incident hypertension. The association between OSA, hyperaldosteronism and hypertension seems to be clearer nowadays. Several clinical studies have indicated that excessive aldosterone levels correlate with the severity of OSA and resistant hypertension.

The blockade of aldosterone reduces the symptoms of OSA [57]. It has been observed that the severity of OSA is greater in patients with hyperaldosteronism and is related to the degree of aldosterone excess. A few reports in the literature suggest the usefulness of spironolactone, a relatively weak diuretic, in resistant hypertension. Some authors hypothesize that hyperaldosteronism worsens OSA by promoting accumulation of fluid within the neck, which leads to increased upper airway resistance [58].

According to the results of most studies, hypertensive patients with OSA are defined as being resistant to therapy when their pressures exceed their goal despite treatment with 3 antihypertensive drugs. Most clinicians have a tendency to treat resistant patients with stronger and stronger agents at higher and higher doses. In contrast, a few recent clinical studies have found that aldosterone blockade with spironolactone in low doses decreases the severity of OSA in patients suffering from resistant hypertension. These patients were treated with spironolactone 25-50 mg daily, which was associated with an almost $50 \%$ reduction of the severity of OSA [59]. Gaddam et al. reported that aldosterone antagonists provide significant additional blood pressure reduction when added to treatment regimens of patients with resistant hypertension and this is generally well tolerated. However, such treatment may predispose patients to hyperkalemia; therefore, biochemical monitoring is necessary, especially in high-risk patients [60].

Results of studies measuring the levels of aldosterone in patients with OSA and the effects

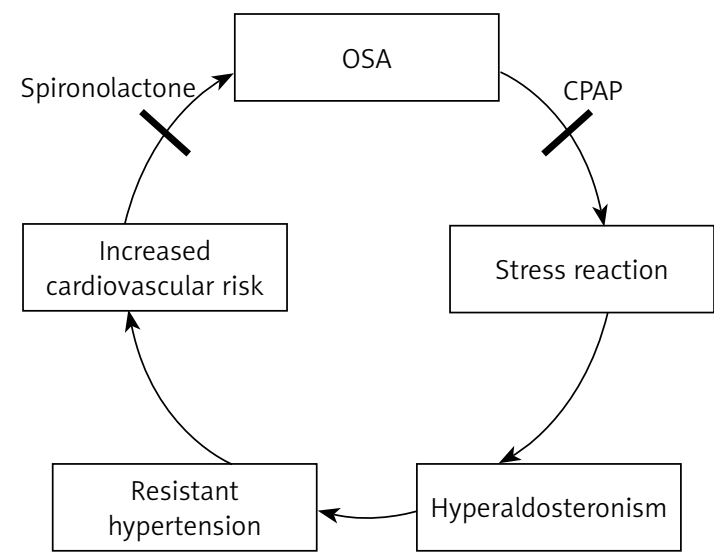

Figure 2. OSA and hyperaldosteronism of CPAP are conflicting. Alajmi et al. performed a meta-analysis of ten randomized controlled trials which included 587 patients with OSA and hypertension. They found that the effects of CPAP were modest and not statistically significant - CPAP reduced systolic blood pressure (SBP) by $3.03 \mathrm{~mm} \mathrm{Hg}$ (95\% Cl: 6.7 to $-0.61 ; p=0.10$ ) and diastolic blood pressure (DBP) by $2.03 \mathrm{~mm} \mathrm{Hg}(95 \% \mathrm{Cl}: 4.1$ to $-0.002 ; p=0.05)$, and the reduction was strongly associated with CPAP compliance [61].

In sharp contrast to these results, the systematic review and meta-analysis of 1000 patients performed by Schein et al. indicated that treatment with CPAP promoted significant reductions in blood pressure in individuals with OSA [62]. On the basis of such differing data, further studies should be performed to evaluate the effects of long-term CPAP and its impact on cardiovascular risk (Figure 2).

\section{Obstructive sleep apnea, insulin resistance and altered glucose metabolism}

Insulin sensitivity in adipocytes and skeletal muscle is impaired by activation of aldosterone-induced mineralocorticoid receptors and by altering potassium levels. Thus, hyperaldosteronism may result in worse glucose tolerance [63]. Barcelo et al. performed a study of 66 patients with OSA (33 with metabolic syndrome and 33 without metabolic syndrome) and 35 controls [64]. The results of the study show that aldosterone levels are strongly associated with the severity of OSA and are higher than in controls.

Significant differences in aldosterone levels were detected between OSA patients with and without metabolic syndrome $(p=0.041)$. They also observed a significant reduction of plasma aldosterone levels in patients under CPAP treatment $(p=0.012)$ [64]. Visceral adiposity and insulin resistance are associated with increased plasma aldosterone and other adrenal steroids that may contribute to cardiovascular diseases in obese non-hypertensive women. Goodfriend et al. observed elevated plasma aldosterone, plasma cortisol and dehydroepiandrosterone sulfate in women. After weight loss, plasma aldosterone was significantly lower and insulin sensitivity higher [65].

Numerous studies have shown that, in patients with moderate-to-severe obstructive sleep apnea, compliant CPAP usage may improve insulin secretion capacity, and reduce leptin, total cholesterol, and low-density lipoprotein levels [66]. Leptin showed a significant relationship with insulin resistance, and this relationship remained after 8 weeks of CPAP therapy [67].

Yang et al. conducted a meta-analysis to evaluate the effects of CPAP on glycemic control and 
insulin resistance in OSA patients. The analysis showed that CPAP significantly improved insulin resistance in non-diabetic patients with moderate to severe OSA, while no significant change in body mass index was detected. Compared with fasting blood glucose at baseline, there was no change in glycemic control with CPAP [68].

On the other hand, Steiropoulos et al. found that night-time hypoxia can affect fasting insulin levels, even in non-diabetic OSA patients, and that long-term CPAP treatment can significantly reduce $\mathrm{HbA}_{1 \mathrm{c}}$ levels, while having no effect on markers of insulin resistance [69]. Further large-scale and controlled studies are needed to estimate possible effects on weight loss and glycemic homeostasis due to longer CPAP treatment.

\section{Obstructive sleep apnea, hypercortisolemia and stress reaction}

Sleep has well-known modulatory effects on hypothalamus-pituitary-adrenal (HPA) axis activity. Sleep onset exerts an inhibitory effect on cortisol secretion, while awakenings and sleep offset are accompanied by intensive cortisol production. Abrupt shifts of the sleep period induce a profound disruption in the daily cortisol rhythm, while sleep deprivation or reduced sleep quality seems to result in a modest but functionally important activation of the axis. Human sleep habits are deeply modified by modern lifestyle. Over recent decades sleep duration has shortened from 8 to $6.5 \mathrm{~h}$, resulting in chronic sleep deprivation. Furthermore, accessory disruption of circadian rhythms is nowadays caused by shift work and traveling across time zones. This results in asynchrony between the master hypothalamic clock and pacemakers in peripheral tissues.

Impairment of HPA axis activity has been described in OSA. Nocturnal awakenings are strongly associated with pulsate cortisol release and autonomic activation [70]. However, the literature lacks agreement regarding the effects of OSA on HPA. Some authors have reported elevated cortisol secretion related with increased catecholamine in patients with OSA, assessed either by $24 \mathrm{~h}$ profiles or by single measurements of evening cortisol levels, together with markers of sympathetic activation and circulating catecholamines after total or partial sleep deprivation [35].

In contrast, in other studies altered HPA axis activity has not been observed, and there has been no significant effect on serum cortisol [71]. Recent studies have identified that disrupted cortisol rhythm leads to coexisting elevated free fatty acid levels and decreased leptin levels. It seems to be possible that endocrine mechanisms contributing to the elicitation of insulin resistance and $\beta$-cell dysfunction in shift workers affect cir- cadian disruption [72]. Untreated hyperactivity of the HPA axis in OSA leads to the development or worsening of metabolic syndrome.

To our knowledge, several studies have focused on the effects of CPAP on cortisol production. Some authors have reported that CPAP therapy does not reduce cortisol plasma levels. According to the meta-analysis performed by Tomfohr et al., the available studies do not provide clear evidence that OSA is associated with alterations in cortisol levels or that treatment with CPAP changes cortisol levels [73]. In contrast, other research has revealed that untreated compared to treated OSA is associated with marked disturbances in ACTH and cortisol secretory dynamics [74]. Additionally, it has been demonstrated that continuous positive airway pressure therapy decreases evening cortisol concentrations in patients with severe obstructive sleep apnea [75] and that this observed effect may be clinically used in the treatment of OSA, particularly in patients with an increased risk of cardiovascular events.

\section{Coexistence of obstructive sleep apnea and inflammation response}

Epidemiological studies suggest that sleep, especially deep sleep, has an inhibitory influence on the HPA axis, while sleeplessness can lead to induction of HPA axis activity. Insomnia causes a 24-hour increase in ACTH and cortisol secretion. Recent studies have demonstrated that sleep deprivation in disorders associated with excessive daytime sleepiness, such as sleep apnea, narcolepsy and idiopathic hypersomnia, provokes an elevation of pro-inflammatory cytokines interleukin (IL)-6 and/or tumor necrosis factor (TNF), whereas daytime napping following a night of total sleep loss appears to be beneficial both for the suppression of IL- 6 secretion and for the improvement of alertness. These findings suggest that the HPA axis stimulates arousal, while IL- 6 and TNF are facultative mediators of excessive daytime sleepiness in humans [76].

Obviously, OSA is a state of inflammation associated with elevated levels of $C$-reactive proteins, IL-6, nuclear factor (NF)- $\mathrm{B}$, TNF- $\alpha$, intercellular adhesion molecule 1 (ICAM-1), vascular cell adhesion molecule 1 (VCAM-1) and E-selectin. Moreover, it is highly probable that oxidative stress induced by nocturnal intermittent hypoxia plays a major role in the formation of reactive oxygen species from leukocytes, the reduction in plasma levels of nitrite, nitrate and antioxidant capacity, and increased lipid peroxidation, all of which are predisposing factors for atherogenesis [77]. This phenomenon has been complemented by biopsy studies demonstrating reduced endothelial nitric oxide synthase expression and increased nitroty- 
rosine immunofluorescence in the vasculature of such patients.

Furthermore, oxidative stress in OSA correlates with markers of cardiovascular disease such as endothelial function, high blood pressure and its consequences such as intima-media thickness and left ventricular concentric remodeling. Deleterious oxidative stress in OSA is believed to be reversible via CPAP therapy. The same effect may hold for antioxidants; however, more studies are needed to clarify this issue [78].

\section{Obstructive sleep apnea and weight control}

The prevalence of OSA among obese patients exceeds $30 \%$, reaching as high as $50-98 \%$ in the morbidly obese population. Obesity is probably the most important risk factor for the development of OSA. Most adults with OSA are overweight (60-90\%). Numerous studies have shown the development or worsening of OSA with increasing weight. Neck circumference seems to give the strongest association with OSA, and waist-hip ratio is a risk factor especially in severe obstructive sleep apnea syndrome. Neck circumference and waist-hip ratio seem to be better indicators of allcause mortality than BMI.

Conversely, OSA may itself predispose individuals to worsening obesity because of sleep deprivation, daytime somnolence, and disrupted metabolism. The main mechanisms linking OSA to obesity are increased sympathetic activation, sleep fragmentation, ineffective sleep, and insulin resistance, potentially leading to diabetes and further aggravation of obesity. Additionally, OSA may be associated with changes in leptin, ghrelin, and orexin levels provoking increased appetite and caloric intake and further exacerbating obesity. Thus, it appears that obesity and OSA form a vicious cycle where each results in a worsening of the other $[79,80]$. These observations lead to the conclusion that satisfactory control of OSA may become a key element in the fight against overweight and obesity.

\section{Conclusions}

Patients with obstructive sleep apnea are at increased risk of endocrinal and metabolic disorders such as osteoporosis, hypogonadism, hyperprolactinemia, hyperaldosteronism and hypercortisolism. These illnesses may lead to serious consequences including shortening of life expectancy [81]. Constantly increasing availability of hormonal tests and tools to diagnose OSA gives us the possibility of early detection of coexisting endocrinal and pulmonary abnormalities, thus giving clinicians the chance of proper treatment in the early phase of those disorders. Interactions between OSA and analyzed entities are complicated and multifaceted, so in the review we underlined vital issues raised in recently published articles. It seems clear that such a modern approach to OSA, which brings implementation of correct therapy, will break the vicious circle of insulin resistance and fertility disorders, and also improve weight control and hypertension. Moreover, recognition and understanding of the interplay between local upper airway dysfunction and its endocrinal consequences enable multidisciplinary care of OSA patients. We believe that our review, by giving insight into novel studies, will facilitate this approach. Due to the epidemic of obesity, proper diagnosis and treatment of OSA have become a priority all over the world. We would like to stress that patients suffering from OSA require complementary therapy which may correct endocrinal changes, improve quality of life and protect from associated morbidity and premature death.

\section{Acknowledgments}

Barbara Bromińska and Marek Ruchała contributed equally to this article.

\section{Conflict of interest}

The authors declare no conflict of interest.

\section{References}

1. Muza RT. Central sleep apnoea - a clinical review. J Thorac Dis 2015; 7: 930-7.

2. Hukins CA. Obstructive sleep apnea - management update. Neuropsychiatr Dis Treat 2006; 2: 309-26.

3. Young T, Palta M, Dempsey J, et al. Burden of sleep apnea: rationale, design, and major findings of the Wisconsin Sleep Cohort study. WMJ 2009; 108: 246-9.

4. Swanson CM, Shea SA, Stone KL, et al. Obstructive sleep apnea and metabolic bone disease: insights into the relationship between bone and sleep. J Bone Miner Res 2015; 30: 199-211.

5. Gonnissen HK, Hulshof T, Westerterp-Plantenga MS. Chronobiology, endocrinology, and energy- and food-reward homeostasis. Obes Rev 2013; 14: 405-16.

6. Tomiyama H, Okazaki R, Inoue D, et al. Link between obstructive sleep apnea and increased bone resorption in men. Osteoporos Int 2008; 19: 1185-92.

7. Cauley JA, Danielson ME, Boudreau RM, et al. Inflammatory markers and incident fracture risk in older men and women: the Health Aging and Body Composition Study. J Bone Miner Res 2007; 22: 1088-95.

8. Wang Q, Wu Q, Feng J, Sun X. Obstructive sleep apnea and endothelial progenitor cells. Patient Prefer Adherence 2013; 7: 1077-90.

9. Dobutovic B, Sudar E, Tepavcevic S, et al. Effects of ghrelin on protein expression of antioxidative enzymes and iNOS in the rat liver. Arch Med Sci 2014; 10: 806-16.

10. Abogresha NM, Greish SM, Abdelaziz EZ, Khalil WF. Remote effect of kidney ischemia-reperfusion injury on pancreas: role of oxidative stress and mitochondrial apoptosis. Arch Med Sci 2016; 12: 252-62.

11. Cofta S, Wysocka E, Michalak S, Piorunek T, Batura-Gabryel H, Torlinski L. Endothelium-derived markers and 
antioxidant status in the blood of obstructive sleep apnea males. Eur J Med Res 2009; 14 Suppl. 4: 49-52.

12. Sheweita SA, Khoshhal KI. Calcium metabolism and oxidative stress in bone fractures: role of antioxidants. Curr Drug Metab 2007; 8: 519-25.

13. Begenik H, Aslan M, Dulger AC, et al. Serum leptin levels in gastric cancer patients and the relationship with in sulin resistance. Arch Med Sci 2015; 11: 346-52.

14. Klok MD, Jakobsdottir S, Drent ML. The role of leptin and ghrelin in the regulation of food intake and body weight in humans: a review. Obes Rev 2007; 8: 21-34.

15. Zhang P, Liu J, Long S, Xie X, Guo Y. Association between continuous positive airway pressure and changes in serum leptin in patients with obstructive sleep apnoea: a meta-analysis. Sleep Breath 2014; 18: 695-702.

16. Amstrup AK, Sikjaer T, Mosekilde L, Rejnmark L. Melatonin and the skeleton. Osteoporos Int 2013; 24: 2919-27.

17. Amstrup AK, Sikjaer T, Heickendorff L, Mosekilde L, Rejnmark L. Melatonin improves bone mineral density at the femoral neck in postmenopausal women with osteopenia: a randomized controlled trial. J Pineal Res 2015; 59 221-9.

18. Zirlik S, Hildner KM, Targosz A, et al. Melatonin and omentin: influence factors in the obstructive sleep apnoea syndrome? J Physiol Pharmacol 2013; 64: 353-60.

19. Bozkurt NC, Cakal E, Sahin M, Ozkaya EC, Firat H, Delibasi T. The relation of serum 25-hydroxyvitamin-D levels with severity of obstructive sleep apnea and glucose metabolism abnormalities. Endocrine 2012; 41: 518-25.

20. Liguori C, Romigi A, Izzi F, et al. Continuous positive airway pressure treatment increases serum vitamin $D$ levels in male patients with obstructive sleep apnea. J Clin Sleep Med 2015; 11: 603-7.

21. Sforza E, Thomas T, Barthelemy JC, Collet P, Roche F. Obstructive sleep apnea is associated with preserved bone mineral density in healthy elderly subjects. Sleep 2013; 36: 1509-15.

22. Wang TY, Lo YL, Chou PC, et al. Associated bone mineral density and obstructive sleep apnea in chronic obstruc tive pulmonary disease. Int J Chron Obstruct Pulmon Dis 2015; 10: 231-7.

23. Song L, Liang $X$, Zhou Y. Estrogen-mimicking isoflavone genistein prevents bone loss in a rat model of obstructive sleep apnea-hypopnea syndrome. Int J Clin Exp Pathol 2014; 7: 1687-94.

24. Chen YL, Weng SF, Shen YC, et al. Obstructive sleep apnea and risk of osteoporosis: a population-based cohort study in Taiwan. J Clin Endocrinol Metab 2014; 99 : 2441-7.

25. Cauley JA, Blackwell TL, Redline S, et al. Hypoxia during sleep and the risk of falls and fractures in older men: the Osteoporotic Fractures in Men Sleep Study. J Am Geriatr Soc 2014; 62: 1853-9.

26. Behan M, Wenninger JM. Sex steroidal hormones and respiratory control. Respir Physiol Neurobiol 2008; 164 213-21.

27. Potvin C, Rossignol O, Uppari N, Dallongeville A, Bairam A, Joseph $V$. Reduced hypoxic ventilatory response in newborn mice knocked-out for the progesterone receptor. Exp Physiol 2014; 99: 1523-37.

28. Driver HS, McLean H, Kumar DV, Farr N, Day AG, Fitzpatrick MF. The influence of the menstrual cycle on upper airway resistance and breathing during sleep. Sleep 2005; 28: 449-56.

29. Shahar E, Redline S, Young T, et al. Hormone replacement therapy and sleep-disordered breathing. Am J Respir Crit Care Med 2003; 167: 1186-92.
30. Zabka AG, Mitchell GS, Behan M. Conversion from testosterone to oestradiol is required to modulate respiratory long-term facilitation in male rats. J Physiol 2006; 576: 903-12.

31. Wittert G. The relationship between sleep disorders and testosterone in men. Asian J Androl 2014; 16: 262-5.

32. Alvarenga TA, Hirotsu C, Mazaro-Costa R, Tufik S, Andersen ML. Impairment of male reproductive function after sleep deprivation. Fertil Steril 2015; 103: 1355-62.e1.

33. Jauch-Chara K, Schmid SM, Hallschmid M, Oltmanns KM, Schultes B. Pituitary-gonadal and pituitary-thyroid axis hormone concentrations before and during a hypoglycemic clamp after sleep deprivation in healthy men. PloS One 2013; 8: e54209.

34. Leproult R, Van Cauter E. Effect of 1 week of sleep restriction on testosterone levels in young healthy men. JAMA 2011; 305: 2173-4.

35. Reynolds AC, Dorrian J, Liu PY, et al. Impact of five nights of sleep restriction on glucose metabolism, leptin and testosterone in young adult men. PloS One 2012; 7 : e41218.

36. Schmid SM, Hallschmid M, Jauch-Chara K, Lehnert H, Schultes B. Sleep timing may modulate the effect of sleep loss on testosterone. Clin Endocrinol (Oxf) 2012; 77: 749-54.

37. Kalmbach DA, Arnedt JT, Pillai V, Ciesla JA. The impact of sleep on female sexual response and behavior: a pilot study. J Sex Med 2015; 12: 1221-32.

38. Luboshitzky R, Aviv A, Hefetz A, et al. Decreased pituitary-gonadal secretion in men with obstructive sleep apnea. J Clin Endocrinol Metab 2002; 87: 3394-8.

39. Gambineri A, Pelusi C, Pasquali R. Testosterone levels in obese male patients with obstructive sleep apnea syndrome: relation to oxygen desaturation, body weight, fat distribution and the metabolic parameters. J Endocrinol Invest 2003; 26: 493-8.

40. Barrett-Connor E, Dam TT, Stone K, Harrison SL, Redline $S$, Orwoll E. The association of testosterone levels with overall sleep quality, sleep architecture, and sleep-disordered breathing. J Clin Endocrinol Metab 2008; 93: 2602-9.

41. Zhang XB, Jiang XT, Du YP, Yuan YT, Chen B. Efficacy of continuous positive airway pressure on testosterone in men with obstructive sleep apnea: a meta-analysis. PloS One 2014; 9: e115033.

42. Bercea RM, Mihaescu T, Cojocaru C, Bjorvatn B. Fatigue and serum testosterone in obstructive sleep apnea patients. Clin Respir J 2015; 9: 342-9.

43. Hanafy HM. Testosterone therapy and obstructive sleep apnea: is there a real connection? J Sex Med 2007; 4: 1241-6.

44. Subramanian S, Bopparaju S, Desai A, Wiggins T, Rambaud C, Surani S. Sexual dysfunction in women with obstructive sleep apnea. Sleep Breath 2010; 14: 59-62.

45. Petersen M, Kristensen E, Berg S, Giraldi A, Midgren B. Sexual function in female patients with obstructive sleep apnea. J Sex Med 2011; 8: 2560-8.

46. Stavaras C, Pastaka C, Papala M, et al. Sexual function in pre- and post-menopausal women with obstructive sleep apnea syndrome. Int J Impot Res 2012; 24: 228-33.

47. Celec P, Mucska I, Ostatnikova D, Hodosy J. Testosterone and estradiol are not affected in male and female patients with obstructive sleep apnea treated with continuous positive airway pressure. J Endocrinol Invest 2014; 37: 9-12.

48. Vgontzas AN, Legro RS, Bixler EO, Grayev A, Kales A, Chrousos GP. Polycystic ovary syndrome is associated 
with obstructive sleep apnea and daytime sleepiness: role of insulin resistance. J Clin Endocrinol Metab 2001; 86: $517-20$

49. Mokhlesi B, Scoccia B, Mazzone T, Sam S. Risk of obstructive sleep apnea in obese and nonobese women with polycystic ovary syndrome and healthy reproductively normal women. Fertil Steril 2012; 97: 786-91.

50. Kopelman PG. Physiopathology of prolactin secretion in obesity. Int J Obes Relat Metab Disord 2000; 24 Suppl 2: S104-8.

51. Onem K, Erol B, Sanli O, et al. Is sexual dysfunction in women with obstructive sleep apnea-hypopnea syndrome associated with the severity of the disease? A pilot study. J Sex Med 2008; 5: 2600-9.

52. Macrea MM, Martin TJ, Zagrean L. Infertility and obstruc tive sleep apnea: the effect of continuous positive airway pressure therapy on serum prolactin levels. Sleep Breath 2010; 14: 253-7.

53. Bratel T, Wennlund A, Carlstrom K. Pituitary reactivity, androgens and catecholamines in obstructive sleep apnoea. Effects of continuous positive airway pressure treatment (CPAP). Respir Med 1999; 93: 1-7.

54. Meston N, Davies RJ, Mullins R, Jenkinson C, Wass JA Stradling JR. Endocrine effects of nasal continuous positive airway pressure in male patients with obstructive sleep apnoea. J Intern Med 2003; 254: 447-54.

55. Spiegel K, Follenius M, Krieger J, Sforza E, Brandenberger G. Prolactin secretion during sleep in obstructive sleep apnoea patients. J Sleep Res 1995; 4: 56-62.

56. Lee EK, Gutcher ST, Douglass AB. Is sleep-disordered breathing associated with miscarriages? An emerging hypothesis. Med Hypoth 2014; 82: 481-5.

57. Dudenbostel T, Calhoun DA. Resistant hypertension, obstructive sleep apnoea and aldosterone. J Hum Hypertens 2012; 26: 281-7.

58. Gonzaga CC, Gaddam KK, Ahmed MI, et al. Severity of obstructive sleep apnea is related to aldosterone status in subjects with resistant hypertension. J Clin Sleep Med 2010; 6: 363-8.

59. Calhoun DA, Nishizaka MK, Zaman MA, Harding SM. Aldosterone excretion among subjects with resistant hypertension and symptoms of sleep apnea. Chest 2004; 125: 112-7.

60. Gaddam KK, Pratt-Ubunama MN, Calhoun DA. Aldosterone antagonists: effective add-on therapy for the treatment of resistant hypertension. Expert Rev Cardiovasc Ther 2006; 4: 353-9.

61. Alajmi M, Mulgrew AT, Fox J, et al. Impact of continuous positive airway pressure therapy on blood pressure in patients with obstructive sleep apnea hypopnea: a meta-analysis of randomized controlled trials. Lung 2007; 185: 67-72.

62. Schein AS, Kerkhoff AC, Coronel CC, Plentz RD, Sbruzzi G. Continuous positive airway pressure reduces blood pressure in patients with obstructive sleep apnea: a systematic review and meta-analysis with 1000 patients. J Hypertens 2014; 32: 1762-73.

63. Luther JM. Effects of aldosterone on insulin sensitivity and secretion. Steroids 2014; 91: 54-60.

64. Barcelo A, Pierola J, Esquinas C, et al. Relationship between aldosterone and the metabolic syndrome in patients with obstructive sleep apnea hypopnea syndrome: effect of continuous positive airway pressure treatment. PloS One 2014; 9: e84362.

65. Goodfriend TL, Kelley DE, Goodpaster BH, Winters SJ. Visceral obesity and insulin resistance are associated with plasma aldosterone levels in women. Obes Res 1999; 7: 355-62
66. Chen L, Pei JH, Chen HM. Effects of continuous positive airway pressure treatment on glycaemic control and insulin sensitivity in patients with obstructive sleep apnoea and type 2 diabetes: a meta-analysis. Arch Med Sci 2014; 10: 637-42

67. Cuhadaroglu C, Utkusavas A, Ozturk L, Salman S, Ece T. Effects of nasal CPAP treatment on insulin resistance, lipid profile, and plasma leptin in sleep apnea. Lung 2009; 187: 75-81.

68. Yang D, Liu Z, Yang H, Luo Q. Effects of continuous positive airway pressure on glycemic control and insulin resistance in patients with obstructive sleep apnea: a meta-analysis. Sleep Breath 2013; 17: 33-8.

69. Steiropoulos P, Papanas N, Nena E, et al. Markers of glycemic control and insulin resistance in non-diabetic patients with obstructive sleep apnea hypopnea syndrome: does adherence to CPAP treatment improve glycemic control? Sleep Med 2009; 10: 887-91.

70. Ekstedt M, Akerstedt T, Soderstrom M. Microarousals during sleep are associated with increased levels of lipids, cortisol, and blood pressure. Psychosom Med 2004, 66: 925-31.

71. Grunstein RR, Handelsman DJ, Lawrence SJ, Blackwell C, Caterson ID, Sullivan CE. Neuroendocrine dysfunction in sleep apnea: reversal by continuous positive airways pressure therapy. J Clin Endocrinol Metab 1989; 68: 352-8.

72. Lee J, Moulik M, Fang Z, et al. Bmal1 and beta-cell clock are required for adaptation to circadian disruption, and their loss of function leads to oxidative stress-induced beta-cell failure in mice. Mol Cell Biol 2013; 33: 2327-38.

73. Tomfohr LM, Edwards KM, Dimsdale JE. Is obstructive sleep apnea associated with cortisol levels? A systematic review of the research evidence. Sleep Med Rev 2012 16: 243-9.

74. Henley DE, Russell GM, Douthwaite JA, et al. Hypothalamic-pituitary-adrenal axis activation in obstructive sleep apnea: the effect of continuous positive airway pressure therapy. J Clin Endocrinol Metab 2009; 94: 4234-42.

75. Schmoller A, Eberhardt F, Jauch-Chara K, et al. Continuous positive airway pressure therapy decreases evening cortisol concentrations in patients with severe obstructive sleep apnea. Metabolism 2009; 58: 848-53.

76. Vgontzas AN, Chrousos GP. Sleep, the hypothalamic-pituitary-adrenal axis, and cytokines: multiple interactions and disturbances in sleep disorders. Endocrinol Metab Clin North Am 2002; 31: 15-36.

77. Ramar K, Caples SM. Vascular changes, cardiovascular disease and obstructive sleep apnea. Future Cardiol 2011; 7: 241-9.

78. Eisele HJ, Markart P, Schulz R. Obstructive sleep apnea, oxidative stress, and cardiovascular disease: evidence from human studies. Oxid Med Cell Longev 2015; 2015: 608438.

79. Pillar G, Shehadeh N. Abdominal fat and sleep apnea: the chicken or the egg? Diabetes Care 2008; 31 Suppl. 2: S303-9.

80. Krzysztoszek J, Wierzejska E, Zielinska A. Obesity. An analysis of epidemiological and prognostic research. Arch Med Sci 2015; 11: 24-33.

81. Destors M, Tamisier R, Baguet JP, Levy P, Pepin JL. Cardiovascular morbidity associated with obstructive sleep apnea syndrome. Rev Mal Respir 2014; 31: 375-85. 